\title{
The effect of a theory- informed behavior change techniques incorporated into a pedometer-based intervention on physical activity and weight of women with polycystic ovarian syndrome: a randomized controlled trial
}

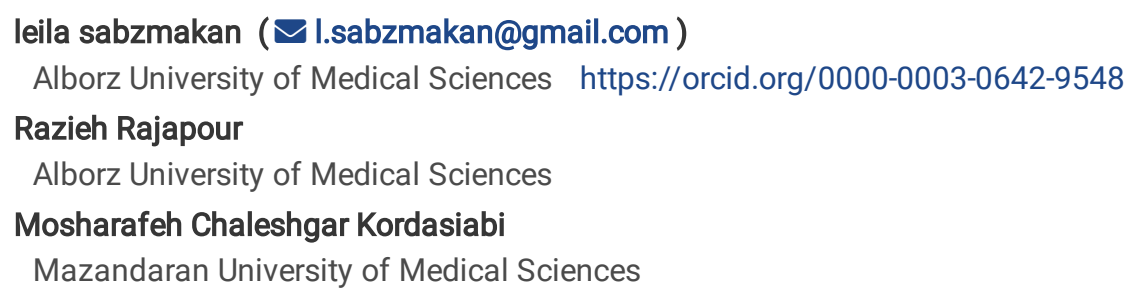

Research

Keywords: Physical activity, Polycystic Ovarian Syndrome, Theoretical framework, Behavior change techniques, Pedometer

Posted Date: February 17th, 2020

DOI: https://doi.org/10.21203/rs.2.23725/v1

License: (1) This work is licensed under a Creative Commons Attribution 4.0 International License. Read Full License 


\section{Abstract}

Background : Increasing physical activity plays an important role in the promotion of the quality of life of women with polycystic ovarian syndrome (PCOS). This study examined the impact of a theory-informed behavior change techniques intervention in conjunction with a pedometer-based intervention on physical activity and weight of women with PCOS.

Method : This research is an unmasked controlled trial study. 88 women with PCOS referred to Mahdieh hospital Tehran, Iran and met the inclusion criteria were assigned by random assignment method to the intervention (12-week pedometer-based intervention plus a theoryinformed behavior change intervention) and the comparison (12-week pedometer-based intervention) group. The primary outcome was physical activity that was measured in two ways. Step-counts were measured using pedometer, and self-reported physical activity was measured via the international physical activity questionnaire. The secondary outcomes were predisposing, enabling, and reinforcing factors that were measured through a psychometric self-administered questionnaire. Weight was also measured as a secondary outcome. All outcomes were measured at baseline and after 12 weeks. Data were analyzed using STATA software version 13. Data analysis was performed on an intention to treat basis using Independent T-Test, ANOVA ANCOVA test, Repeated Measure ANOVA, and post-hoc analysis.

Results : After adjusted mean, significant increases with a strong and moderate effect size were found in the intervention group relative to the comparison group for step-counts $(P=0.000, d=2.01)$, walking $(P=0.000, d=0.92)$, predisposing factors $(P=0.000, d=1.05)$, enabling and reinforcing factors $(P<0.05, d=0.54-0.60)$. Significant decrease was found in the intervention group compared to the comparison group for weight $(P=0.005)$ with a moderate effect size $(d=0.66)$.

Conclusion : This pedometer-based intervention that applied behavior change techniques on the basis of predisposing, enabling, and reinforcing factors of the PRECEDE framework was affective to enhance physical activity and diminish the weight of women with PCOS. Therefore, it is suggested to incorporate theory-informed behavior change techniques into pedometer-based interventions for promoting physical activity.

Trial registration : Iranian Registry of Clinical Trials IRCT20161116030923N3.

Keywords: Physical activity, Polycystic Ovarian Syndrome, Theoretical framework, Behavior change techniques, Pedometer

\section{Introduction}

Polycystic ovarian syndrome (PCOS) is one of the most common disorders of endocrine glands, affecting $6-12 \%$ of women during the reproductive age(1). The syndrome is first characterized by ovarian dysfunction and hyperandrogenismand, and it is followed by a wide range of complications, such as amenorrhea, anovulatory, infertility, hirsutism, metabolic disorder (insulin resistance and the increased risk of type 2 diabetes and cardiovascular disease), mental problems, some cancers, and decreased quality of life(2). The prevalence of obesity and overweight among women with this syndrome is $40-80 \%(2,3)$. In an Iranian national study, the prevalence of PCOS has been reported to be $14.6 \%$ (4). PCOS imposes a considerable cost on the healthcare system. In the United States, \$36.4 billion is spent on PCOS and related complications, and more than $40 \%$ of this cost is dedicated to the treatment of reproductive disorders (infertility and menstrual dysfunction), and other $40 \%$ is dedicated to the diabetes related to PCOS (5). Therefore, treatment and effective interventions are needed to reduce the burden of this disease and improve health outcomes among women with PCOS.

Nowadays, lifestyle modification including promoting physical activity(PA) and healthy eating has been introduced as the first line treatment for patients with PCOS(4). Evidence-based guidelines have suggested lifestyle modification as a non-pharmaceutical treatment for PCOS $(6,7)$. According to the priority and importance of PA among PCOS women, it is necessary to identify the factors influencing PA and modify them(8). Moreover, it has been reported that women with PCOS have a higher level of depression compare with healthy women; therefore, PA seems efficacious to reduce the symptoms of depression among these patients(8).

There are limited studies on PA among women with PCOS, and the results are inconsistent. In some studies, the level of PA among these women was reported to be less than healthy women, while other studies did not show a significant difference in PA between women with and without PCOS(9). The obstacles such as fatigue, lack of time, lack of self-confidence to maintain PA, fear of injury, and physical limitations have reported for doing PA(8). The use of pedometer as a useful motivational tool to increase walking has been confirmed(10, 11). A study (12) reported that achieving 10,000 steps/day is associated with promoting PA and important health outcomes. In addition, a systematic review study (10) indicated that pedometer users increased their PA and it led to significantly decrease of weight, body mass index, and blood pressure. 
On the other hand, PA is a multi-factorial behavior is affected by intrapersonal, interpersonal, environmental, and social factors(13). According to Bartholomew et al.(14), theories can be used to identify the factors that might influence a behavior, and then modify these factors using behavioral change techniques (BCTs). PA interventions were carried out based on the theory of planned behavior, socialcognitive theory, and the trans-theoretical model along with pedometer; however, there were limitations for each of these theories, for example, these theories emphasized on individual factors-versus social and structural factors.

The use of the PRECEDE (Predisposing, Reinforcing, and Enabling Constructs in Educational Diagnosis and Evaluation) framework has been introduced as a logical model for identifying health problem, its behavioral and environmental factors, as well as the determinants associated with these factors $(14,15)$. On the basis of the PRECEDE model, individual, social, and structural factors that influence a behavior can be identified, and these factors can be modified by using BCTs(14). A taxonomy of BCTs was developed by Michie et al. by using expert consensus approach(16). A BCT is defined as "an observable, replicable, and irreducible component of an intervention designed to alter or redirect causal processes that regulate behavior, that is, a technique is proposed to be an 'active ingredient" (16). The PRECEDE framework was used in some researches to identify the factors related to a behavior $(17,18)$, and the relevant BCTs were suggested based on the determinants of that behavior $(19,20)$. No research to date, to our knowledge, has been published using BCTs based on a theoretical framework to modify the factors associated with PA among PCOS women. This study was conducted with the aim of the effect of a pedometer-based intervention, incorporating BCTs on the basis of predisposing, enabling, and reinforcing factors of the PRECEDE framework, on step-counts, PA and weight of women with PSCO.

\section{Method}

\section{Study design}

The present study was carried out as an unmasked controlled trial to examine the effect of a pedometer-based intervention, in conjunction with BCTs on the basis of a theoretical framework on step-counts, PA and weight of women with PCOS referral to Mahdieh hospital Tehran, Iran, in 2017. To design and write this article, Consort 2010 checklists and flow diagram were used(21). Sampling was performed by random assignment method with 1:1 ratio and permuted block randomization. In this method, because of the two groups several blocks with letters A and B were identified. The size of each block was 4 . The sample size was divided by the size of the block to obtain the number of blocks. Since the sample size was 88,22 blocks were estimated. Each block specifies the status of 4 clients. The website www.randomization.com (22) was used to build the randomization sequence. To conceal generated lists, the lists with letters $A$ and $B$ in the specified order were put in thick envelopes by first author, who did not involve in the processes of intervention, and then the serial numbers were written on all envelopes. According to the mean and standard deviation of steps-counts in Becker and colleagues' study(23) as well as G-power software, the sample size was calculated. In G-power software, using F family test, repeated measure ANOVA test, the effect size= $0.35, \beta=0.90, a=0.05$, by 12 times pointes measuring step-counts, and correlation coefficient $=0.7$, the sample size was calculated 33 in each group. Considering $30 \%$ dropout rate, the sample size reached 44 people in each group at the beginning of the study.

\section{Participants}

The study populations were women with PCOS referral to Mahdieh hospital in Tehran. The inclusion criteria were PCOS according to the gynecologist diagnosis, age range of 18-40 years, written and oral consent to participate in study, literacy, lack of disability and movement limitation, lack of mental disorder, and lack of a chronic disease not allowed by the physician to perform PA such as advanced diabetes and cardiovascular disease, etc. The exclusion criteria included pregnancy and lactation, hormonal drugs use, using infertility treatments methods, and smoking.

\section{Measurement of outcome}

\section{Primary outcome}

The primary outcome was PA that was measured in two ways. Objective PA or step-counts was measured using the Rossmax PA-S20 pedometer, a two-by-two-size electronic tool that is attached to the belt and displays step-counts. The validity and reliability of pedometer for measuring step-counts were supported (24). Subjective or self-reported PA was measured using the International Physical Activity Questionnaire (IPAQ) long form. The IPAQ is used as a standardized instrument to estimate PA among populations from different countries and cultural contexts.(25) It involves 7-day recall of PA. This questionnaire consists of 27 items and assesses the time spent in walking, 
moderate and vigorous activity within the domains of work, leisure time, transportation, domestic and gardening(25). Total scores for PA were calculated based on metabolic equivalents (MET) minutes per week and PA levels were classified as low (MET $\geq 600)$, moderate (600 $<$ MET < 3000) and vigorous (MET > 3000)(25). In 2003, Craig et al. analyzed the validity and reliability of the IPAQ in 12 countries (26). Baghiani Moghaddam and colleagues also investigated the validity and reliability of the Persian version of IPAQ among Iranian women(27).

\section{Secondary outcomes}

Predisposing, enabling, and reinforcing factors which influence PA behavior were secondary outcomes of current study. According to the educational phase of the PRECEFE framework, predisposing, enabling, and reinforcing factors play an important role in the appearance of a behavior(15). Predisposing factors come prior to behaviors and include knowledge, attitudes, beliefs, existing skills, self-efficacy, and personal preferences that facilitate or prevent the motivation for change $(15,28)$. Reinforcing factors are followed by a behavior providing continuous reward and motivation for the emergence or repetition of a behavior. For example, they include social support, significant others, peer influence, positive/negative behavioral outcomes, and reward by others $(15,28)$. The factors that are antecedents to behavior and allow a motivation or environmental policy to be achieved are called enabling factors; such as the existence and access to resources and services, new skills, programs, rules and regulations $(15,28)$. In this study, to measure predisposing, reinforcing, and enabling factors, a selfadministered questionnaire was designed based on the previous researches $(17,18)$. This questionnaire consisted of two parts. The first part included 10 demographic questions and the second part consisted of the questions related to predisposing factors (6 knowledge items, 7 attitude items, and, 9 self-efficacy items), reinforcing factors (5 social support items and 4 behavioral outcomes items), and enabling factors ( 2 items of new skills, 2 items of resources and 3 items of rules and policies). The items of knowledge, enabling factors, and behavioral outcomes were with a 3-point scale from 1=yes, 2=I do not know/somewhat and 3=no and other items rated with 5-point Likert scale from 1 (strongly disagree) to 5 (strongly agree). After developing the questionnaire, its validity and reliability was measured. To determine face validity, the items with impact score equal or greater than 1.5 were considered as reasonable by 10 participants with the same characteristics of the target population (29). The content validity index (CVI) and content validity ratio (CVR) of items were also investigated by 10 experts in health education and midwife. The score of CVR was calculated according to the necessity of each item, and a score equal or higher than 0.62 on the basis of the Lawshe table was envisaged a good content validity. To determine the CVI, the correlation criteria, clarity, and simplicity of each item were computed and a score equal to or higher than 0.79 was considered as reasonable (29). In addition to validity, the reliability of the questionnaire was assessed through internal consistency(29) by 15 participants with the same characteristics of the target population. Cronbach's Alpha coefficient for all constructs ranged from 0.71-0.91. Weight as other secondary outcome of this study was also calculated through an accurate scale in kilogram. All questionnaires were completed by interview and all outcomes were measured by a person outside the research team.

\section{Ethical considerations}

The study protocol was approved by the ethics committee of the Alborz University of Medical Sciences 2016 (approval No. Abzums. Rec.1395.23). All women participating in the study were informed about the objectives of research and assured about the confidentiality of their information. In addition, informed written consent forms were completed by participants in both groups. This study protocol was registered in the Iranian Registry of Clinical Trials as IRCT20161116030923N3. In order to comply with ethical consideration, the comparison group was benefited from theory-based intervention at the end of the study.

\section{Theory-based educational intervention}

A pedometer (Rossmax PA-S20 Pedometer), a calendar to record daily steps, an educational video and a pamphlet about using pedometer, and face-to-face training were given to all participants. Both intervention and comparison groups participated in 12-week pedometer-based walking program. All participants were told not to consider step-counts in the first two weeks and to record their daily steps in the calendar after two weeks and report it to the educator or a team leader at the end of per week. At the beginning of the study, the questionnaires were completed by the eligible individuals in both groups and their weight was also measured. In addition to 12-week pedometer-based walking program, the patients in the intervention group participated in four sessions of behavior change intervention based on predisposing, enabling and reinforcing factors. Since it was not possible to simulate educational intervention for both groups, an unmasked trial was performed. The training sessions were held once a week for 60 minutes. The patients in the intervention group were divided into four groups and a team leader was assigned for each group to timely announce training sessions and receive step-counts. For this purpose, a Telegram Group as "Increasing Step-counts by using Pedometer" was created and all individuals in the four groups joined the network. 
First, on the basis of the PRECEDE framework, the factors associated with PA were identified in three categories of the predisposing, enabling, and reinforcing factors, then, according to the taxonomy table of BCTs $(14,16,30)$, the relevant BCTs were selected for each factor. In predisposing category, a purpose was to increase patients' knowledge about using the pedometer, the PA pyramid, and the importance of regular PA in controlling and reducing the complications of PCOS. Other purposes in this category were positive attitude towards increasing step-counts, and enhancing patients' self-efficacy to increase step-counts. The consciousness raising technique was used in predisposing category to increase patients' awareness. In addition, on Telegram Group, the video and the pamphlet of working with pedometer, the pamphlet of PCOS and the PA pyramid were shared as cues to increase patients' awareness. Repeated measure technique was used for modifying attitude such that participants in training sessions and on Telegram Group were repeatedly exposed to the message of increasing step-counts by 10,000 steps / day. The participants were encouraged to increase step-counts to 10,000 daily steps by using goal setting method. Set graded tasks method was also used to modify self-efficacy for increasing step-counts. To this end, the complex and difficult behavior of 10,000 daily steps was divided into smaller and simpler tasks. This enhanced the understanding and perception of patients that PA behavior could be performed without difficulty. For this purpose, it was explained that every 15 minutes of walking could increase step-counts to 3200-3800 steps. Another technique for increasing self-efficacy in current study was self-monitoring. To this end, the patients was given a weekly calendar, and they were asked to record their steps in the calendar and report it to team leader or the educator through Telegram Group at the end of the week. Also, the patients were contracted to increase step-counts to 10,000 daily steps by using the commitment technique, and the patients committed to walk 10,000 steps /day.

In enabling category, the purpose was to promote the new skills, availability and accessibility of resources and programs to increase stepcounts. Guide practice technique was used to enhance the skill of using the pedometer. The educator trained the patients on how to use the pedometer via face-to-face method, and then they were asked to set up the pedometer on their waist and walk a few steps, and read stepcounts on the pedometer. The resources such as the pedometers, the pamphlets, calendar, the educational videos, informative and persuasive massages to increase steps, Telegram Group, training sessions, individual consultations, group discussions and 12-week followup were provided for the patients. Some structural barriers to PA were mentioned by the participants such as insecurity of parks and sidewalks for walking, polluted air, lack of gyms in the apartments, the distance from the gym, and lack of easy access to the gyms. Planning coping responses technique was used to overcome structural barriers associated with PA. The patients in group discussions identified the barriers associated with PA and reaching 10,000 steps /day and by using brainstorming technique suggested solutions for coping with these obstacles.

In reinforcing category, the purpose was to enhance social support and behavioral outcome in order to increase step-counts. The importance of networking was taught as a way to expand social support. The patients were advised to use the company of their family members or friends to increase their step-counts. They were also told that encouraging each other for walking and doing it in a group is more enjoyable and encouraging. The social comparison technique was used to create a competitive group in increasing step-counts. In addition, reinforcement technique was applied for increasing step-counts. For this purpose, the women who reported more steps were encouraged and rewarded in training sessions. The direct experience and feedback method were used to improve behavioral outcome, meaning that, in training sessions, the women in the intervention group expressed their feelings and status by increasing step-counts and the educator also gave them positive and negative feedback on their performance.

\section{Data analysis}

After data collection, data were entered into SPSS.19 software. In the primary analysis, PA variable and its levels such as low, moderate, vigorous, and total PA, as well as also predisposing, enabling, and reinforcing factors were created by using of the collected data. To assess the normality of data, central tendency, histogram, and Shapiro-Wilk test were used. In case of non-normal distribution of variables, logtransformation was used. In current study, log-transformation was used to make the non-normal distribution data of the levels of PA and weight closely to the normal distribution. In the primary analysis, STATA software version 13 was used to analyze the objectives and assumptions of the study. Independent t-test, ANOVA ANCOVA and Repeated Measure ANOVA were applied for primary analysis. There was a sphericity presumption or reliability of the correlation at time points. Post-hoc analysis was also used for secondary analysis. To assess the relationship power, Cohen's d effect size was used. The effect size classification based on Cohen's $d$ is interpreted as non- effective areas for the value of 0.1 , small effect for $0.2-0.4$, moderate effect for $0.5-0.7$, and strong effect for 0.8 and above(31).

\section{Results}

\section{Participants}


Out of 102 referrals, 88 met the inclusion criteria and provided baseline measurements of pedometer step-counts, IPAQ, the factors influencing PA based on the PRECEDE framework and weight. Each group consisted of 44 patients. Figure 1 displays the flow diagram of participants through the study. As shown in Figure 1, 5 patients in the intervention group and 9 patients in the comparison group dropped out from the study between baseline and week 12 . The rate of drop out in the intervention group was approximately $10 \%$ and in the comparison group was approximately $19 \%$. Thus, 39 females in the intervention group and 35 females in the comparison group completed the study, and whose data were used in the analysis. Data analysis was performed on an intention to treat basis where all participants were considered in the statistical analysis. Table 1 displays the baseline information of both intervention and comparison groups.

Table 1

Baseline characteristics of women with PCOS

\begin{tabular}{|lll|}
\hline Characteristics & $\begin{array}{l}\text { Intervention group } \\
(\mathbf{N}=39)\end{array}$ & $\begin{array}{l}\text { Comparison group } \\
(\mathbf{N}=35)\end{array}$ \\
\hline Age-year & $27.97 \pm 4.72$ & $27.54 \pm 4.98$ \\
\hline $\begin{array}{l}\text { Marital status- no. (\%) } \\
\text { Single }\end{array}$ & $16(41 \%)$ & $25(71 \%)$ \\
\hline Married & $22(56.4 \%)$ & $9(25 \%)$ \\
\hline Divorce & $1(2.6 \%)$ & $1(2.9 \%)$ \\
\hline $\begin{array}{l}\text { Education- no. (\%) } \\
\text { Primary education }\end{array}$ & $5(12.8 \%)$ & $5(14.3 \%)$ \\
\hline Secondary education & $20(51.3 \%)$ & $19(54.3 \%)$ \\
\hline Post-secondary education & $6(15.4 \%)$ & $3(8.6 \%)$ \\
\hline University education & $8(20.5 \%)$ & $8(22.8 \%)$ \\
\hline Job- no. (\%) & $15(28.5 \%)$ & $14(40 \%)$ \\
\hline Housewife & $16(41)$ & $12(34.3)$ \\
\hline Employed & $8(20.5 \%)$ & $9(25.7 \%)$ \\
\hline Unemployed & & \\
\hline
\end{tabular}

\section{Physical Activity}

\section{Step counts}

Figure 2 illustrates the mean steps/week for both groups over 12 weeks. As shown in this figure, the mean step-counts at time points of the first week to week 12 was not equal between both groups and the two lines were not parallel. Repeated Measure ANOVA test indicated a significant interaction was found between groups and time in terms of step-counts. There was no significant difference between the two groups in the time points of the first week until the fourth week, but the mean step-counts was significantly different between the two groups from week 5 to week 12.

The ANOVA ANCOVA model and the factor variable (groups) showed significant effects $(P<0.05)$ in terms of step-counts, however the covariance variable, namely the mean score of step-counts before intervention, did not show a significant effect $(P=0.06)$. The ANOVA ANCOVA test also found a significant difference in the mean of step-counts weekly between the two groups with a strong effect size ( $\mathrm{t}=$ 9.03, $p<0.001, d=2.10$ ). As shown in Table 2, after adjusted mean, there was no difference between crude and adjusted mean of stepcounts $(t=8.54, p<0.001, d=2)$. 
Table 2

The effect 12 weeks pedometer-based intervention along with theory- informed behavior change techniques on cognitive factors, stepcounts, physical activity and weight of women with PCOS

\begin{tabular}{|c|c|c|c|c|c|c|c|c|}
\hline \multirow[t]{2}{*}{$\begin{array}{l}\text { Variable } \\
\text { (Mean } \pm \text { SD) }\end{array}$} & \multicolumn{2}{|c|}{ Before intervention } & \multicolumn{2}{|c|}{ After intervention } & \multicolumn{2}{|c|}{$\begin{array}{l}\text { After intervention adjusted } \\
\text { for before intervention }\end{array}$} & \multirow[t]{2}{*}{$\begin{array}{l}\text { d Cohen } \\
\text { Cl } 95 \%\end{array}$} & \multirow[t]{2}{*}{$\begin{array}{l}\mathrm{P}- \\
\text { value }\end{array}$} \\
\hline & $\begin{array}{l}\text { Intervention } \\
\text { group }\end{array}$ & $\begin{array}{l}\text { Comparison } \\
\text { group }\end{array}$ & $\begin{array}{l}\text { Intervention } \\
\text { group }\end{array}$ & $\begin{array}{l}\text { Comparison } \\
\text { group }\end{array}$ & $\begin{array}{l}\text { Intervention } \\
\text { group }\end{array}$ & $\begin{array}{l}\text { Comparison } \\
\text { group }\end{array}$ & & \\
\hline Predisposing & $1.09 \pm 7.93$ & $0.86 \pm 8.06$ & $0.72 \pm 9.93$ & $0.75 \pm 9.39$ & $0.57 \pm 9.95$ & $0.57 \pm 9.35$ & $\begin{array}{l}1.05(0.56 \\
1.54)\end{array}$ & 0.000 \\
\hline Reinforcing & $5.49 \pm 1.18$ & $5.43 \pm 0.89$ & $6.94 \pm 0.71$ & $6.51 \pm 0.76$ & $6.94 \pm 0.69$ & $6.52 \pm 0.71$ & $0.60(0.13,1.06)$ & 0.01 \\
\hline Enabling & $1.37 \pm 0.26$ & $1.44 \pm 0.33$ & $1.77 \pm 0.16$ & $1.69 \pm 0.28$ & $1.78 \pm 0.19$ & $1.68 \pm 0.18$ & $0.54(0.07,1)$ & 0.03 \\
\hline Step-counts & $\begin{array}{l}3841.51 \pm \\
360.14\end{array}$ & $\begin{array}{l}3627.49 \pm \\
581.84\end{array}$ & $\begin{array}{l}7507.87 \pm \\
844.21\end{array}$ & $\begin{array}{l}5591.71 \pm \\
981.44\end{array}$ & $\begin{array}{l}7465.73 \pm \\
906.69\end{array}$ & $\begin{array}{l}5638.67 \pm \\
907.92\end{array}$ & $\begin{array}{l}2.01(1.44 \\
2.57)\end{array}$ & 0.000 \\
\hline $\begin{array}{l}\text { Total } \\
\text { Physical } \\
\text { Activity(PA) }\end{array}$ & 1197.08. \pm 2.09 & $\begin{array}{l}1081.88 \pm \\
2.04\end{array}$ & $\begin{array}{l}2400.09 \pm \\
1.56\end{array}$ & $\begin{array}{l}1812.11 \pm \\
2.36\end{array}$ & $\begin{array}{l}2352.99 \pm \\
1.83\end{array}$ & $\begin{array}{l}1852.57 \pm \\
1.83\end{array}$ & $\begin{array}{l}0.39(-0.06 \\
0.85)\end{array}$ & 0.10 \\
\hline Low PA & $491.64 \pm 2.20$ & $\begin{array}{l}483.16 \pm \\
2.35\end{array}$ & $\begin{array}{l}1232.83 \pm \\
1.53\end{array}$ & $\begin{array}{l}774.47 \pm \\
1.94\end{array}$ & $\begin{array}{l}1237.05 \pm \\
1.67\end{array}$ & $\begin{array}{l}776.45 \pm \\
1.66\end{array}$ & $\begin{array}{l}0.92(0.44 \\
1.40)\end{array}$ & 0.000 \\
\hline Moderate PA & $596.64 \pm 2.15$ & $\begin{array}{l}502.07 \pm \\
2.12\end{array}$ & $\begin{array}{l}972.62 \pm \\
1.65\end{array}$ & $\begin{array}{l}695.01 \pm \\
3.02\end{array}$ & $\begin{array}{l}952.47 \pm \\
2.17\end{array}$ & $\begin{array}{l}762.26 \pm \\
2.16\end{array}$ & $\begin{array}{l}0.29(-0.17 \\
0.74)\end{array}$ & 0.22 \\
\hline Vigorous PA & $504.95 \pm 3.74$ & $\begin{array}{l}264.15 \pm \\
3.56\end{array}$ & $656.99 \pm 3$ & $\begin{array}{l}435.20 \pm \\
3.39\end{array}$ & $\begin{array}{l}548.41 \pm \\
0.72\end{array}$ & $\begin{array}{l}457.55 \pm \\
0.81\end{array}$ & $\begin{array}{l}0.24(-0.22 \\
0.69)\end{array}$ & 0.34 \\
\hline Wight (kg) & $66.70 \pm 1.13$ & $62.27 \pm 1.12$ & $\begin{array}{l}62.70 \pm \\
1.13\end{array}$ & $59.99 \pm 1.13$ & $\begin{array}{l}60.71 \pm \\
1.04\end{array}$ & $62.19 \pm 1.04$ & $\begin{array}{l}-0.66(-1.13 \\
-0.19)\end{array}$ & 0.005 \\
\hline
\end{tabular}

\section{Self-reported Physical Activity (IPAQ)}

The ANOVA ANCOVA model and covariance variable revealed significant effects $(P=0.0001)$ in terms of total PA, but it was not significant for groups variable $(P=0.10)$. On the other hand, the ANOVA ANCOVA test did not show a significant difference in the mean total PA between the two groups and a small effect size was observed $(t=1.80, p=0.08, d=0.42)$. After adjusted mean, there was a difference between crude and adjusted mean score of total PA $(t=1.70, p=0.10, d=0.39)$. Total self-reported PA consisted of walking, moderate, and vigorous PA. The ANOVA ANCOVA model and variables of groups and covariance indicated significant effects $(P<0.0001)$ in terms of walking. The ANOVA ANCOVA test also identified a significant difference in the mean of walking between both groups with a strong effect size $(t=3.62, p<0.001, d=0.84)$. After adjusted mean, no difference was observed between crude and adjusted mean of walking score $(t=$ $3.91, p<0.001, d=0.92)$. However, in terms of both moderate and vigorous PA, the ANOVA ANCOVA model and the covariance variable were both significant $(P<0.0001)$, but the variable of groups was not significant $(P>0.05)$. The ANOVA ANCOVA test did not also indicate a significant difference in the mean of both moderate PA $(t=1.71, p=0.09, d=0.40)$ and vigorous PA $(t=0.64, p=0.53, d=0.36)$ between the two groups and a small effect size was observed. After adjusted mean, there was a difference between crude and adjusted mean of moderate PA ( $t=1.24, p=0.22, d=0.29)$ and vigorous PA ( $t=1.01, p=0.34, d=0.24)$. Table 2 displays the effect size, $P$-value, confidence interval; crude and adjusted mean score of walking, moderate, vigorous, as well as total PA.

\section{Cognitive Factors}

In terms of predisposing, reinforcing, and enabling factors, the ANOVA ANCOVA model showed a significant effect (P $<0.0001)$, and the variables of both groups and covariance also indicated significant effects $(P<0.05)$. In addition, the ANOVA ANCOVA test identified a significant difference in the mean of predisposing factor between both groups with a strong effect size $(t=3.13, p=0.003, d=0.73)$. After adjusted mean, there was no difference between crude and adjusted mean score of predisposing factor $(t=4.50, p<0.001, d=1.05)$. Furthermore, the ANOVA ANCOVA test found a significant difference in the mean of reinforcing factor between the two groups with a moderate effect size $(t=2.51, p=0.01, d=0.58)$. After adjusted mean, there was no difference between crude and adjusted mean score of reinforcing factor $(t=2.57, p=0.01, d=0.60)$. Moreover, the ANOVA ANCOVA test did not show a significant difference in the mean of enabling factors between the two groups with a small effect size $(t=1.61, p=0.11, d=0.37)$. After adjusted mean, there was difference 
between crude and adjusted mean score of enabling factor, and a significant difference with a moderate effect size was observed between the two groups $(t=2.22, p=0.03, d=0.54)$ (Table 2$)$.

\section{Weight}

Finally, an independent t-test identified a significant difference in the mean weight between the groups before intervention $(P=0.01)$. The ANOVA ANCOVA model and variables of groups and covariance showed significant effects $(P<0.0001)$. The ANOVA ANCOVA test did not also identify a significant difference in the mean of weight between the two groups and a small effect size was observed $(t=1.57, p=0.12$, $d=0.36)$. After adjusted mean, there was a difference between crude and adjusted mean of weight score that indicated the effect of the covariance variable was considerable, so a significant difference with a moderate effect size was observed between both groups $(t=2.92, p$ $=0.005, \mathrm{~d}=-0.66)($ Table 2).

\section{Discussion}

This controlled trial study was conducted to investigate the effect of a pedometer-based intervention, incorporating BCTs on the basis of the PRECEDE framework, on step-counts, walking, and weight of women with PCOS during 12 weeks. The major finding of this study was that a pedometer-based intervention, in conjunction with BCTs based on a theoretical framework increased step-counts and walking significantly with a strong effect size in the intervention group compared with the comparison group over a period of 12 weeks. In addition, this theorybased intervention could decrease the women' weight in the intervention group significantly with a moderate effect size relative to the comparison group. A meta-analysis study by Gourlan et al (32) suggested that theory-based interventions were significantly effective in the promotion PA. In another meta-analysis by Prestwich et al.(33), reported $56 \%$ of interventions a theory-base and stated of those interventions, $90 \%$ reported no relationship between BCTs and specific theoretical structures. Therefore, selecting BCTs on the basis of the factors influencing PA in theory-informed behavior change interventions would be more effective in promoting PA comparison with nontheoretical interventions or those without BCTs.

In current study, predisposing factors including knowledge, attitude, and self-efficacy significantly increased with a strong effect size in the intervention group compared to the comparison group. The score of knowledge and attitude about PA was almost the same among participants of both groups because they were taught about the importance of PA and had also a positive tendency toward using pedometer. Self-efficacy has been identified the main contributing factor in predisposing category to enhance step-counts and walking. Bandura(28) identified self-efficacy as an important contributor to behavior change. A systematic review \& meta-analysis study(34) reported self-efficacy as an important determinant of PA, and BCTs appeared more affective for modifying it. In current study, self-efficacy was modified using BCTs such as goal setting, setting graded tasks, self-monitoring, and commitment. A meta-regression study (35) was conducted on more than 122 studies and 44000 participants, its findings indicated that self-monitoring was the main predictor of successful behavior change for PA. In addition, a review study concluded that there was a significant relationship between goal setting and increasing PA(36). Breaking a long-term goal into achievable objective goals increases self-efficacy (37). Hence, using of setting graded task technique can be affective on increasing PA and step-counts as well as lose weight. Moreover, in a study by Dal-Hyun et al. (38) was shown that goal commitment was significantly able to increase daily steps among adults.

Using the pedometer by both groups was a motivational and reinforcing tool to increase step-counts. In addition to the pedometer, using BCTs significantly increased reinforcing factors such as social support and behavioral outcomes with moderate effect size in the intervention group compared to the comparison group. The mean score of social support in the intervention group was more than the comparison group; however, behavioral outcome was the most important determinant in reinforcing category that promoted step-counts. Behavioral outcome the same outcome expectation is an important construct of Bandura's social cognitive theory and health promotion model that reflects the positive and negative outcomes of an engaging particular behavior by an individual, and it can predict health behavior(39). The participants in the intervention group because of increasing their step-counts reported happiness, fit shape, lose weight, reduction and remove of the symptoms associated with their illness more than the comparison group. Previous studies confirmed the importance of social support and outcome expectation in PA behavior change $(18,40,41)$. In current study, feedback technique was used to improve behavioral outcome. For this purpose, the participants received positive and negative feedback based on their weekly step-counts. A study by Kramer and Kowatsch(42) indicated that providing feedback on performance enhanced PA.

Enabling factor also significantly increased in the intervention group relative to the comparison group with a moderate effect size. Indeed, the mean score of enabling factor increased in both groups because they benefited from resources such as pedometer-based walking program, educational media, and follow-up. However, the participants in the intervention group attended in group sessions and individual consultations. They also received more educational resources and the messages to increase their step-counts. In addition, they were membership on Telegram Group and received feedback on their performance. Jirathananuwat and Pongpirul(43) in a systematic metareview study confirmed the use of resources and facilities as enabling factors improved PA. Moreover, the participants identified some structural barriers to increase step-counts and PA. Therefore, coping planning technique was used to modify the obstacles. The use of 
problem solving or coping planning techniques to reduce obstacles of PA was approved(40,44). It should be noted that BCTs could not reduce the structural barriers related to PA, but also only increased women's ability to cope with these barriers.

Although pedometer-based walking program increased step-counts in both groups over a 12-week period, using BCTs tailored to the predisposing, reinforcing, and enabling factors significantly increased step-counts in the intervention group with a strong effect size relative to the comparison group. A systematic review study on 26 trial and 8 observational studies showed that using pedometer increased PA to $26.9 \%$ compared to baseline. The results of Lutes et al.'s review (40) indicated that although the use of pedometer was so important to enhance PA, BCTs on the basis of a theoretical framework in designing pedometer- based interventions should be used to promote PA behavior. In current study, using BCTs and pedometer could not significantly increase total PA in the intervention group relative to the comparison group, and its effect size was small. This theory- informed behavior techniques intervention, in conjunction with a pedometerbased intervention, only increased walking significantly with a strong effect size in the intervention group compared to the comparison group. However, no significant effect on moderate and vigorous PA levels was not observed and was also reported a small effect size. The reason can be justified by this fact that using of pedometer increases step-counts and walking, so it has a great effect on walking and may has no great effect on moderate and vigorous PA. The study of Pal et al.(45), showed that using of a pedometer affected on walking, and its impact on moderate and vigorous PA was less.

Finally, although using of pedometer in addition to increasing walking and step-counts could reduce weight in both groups, theory- informed behavior change intervention along with pedometer-bases walking decreased the weight of participants in the intervention group significantly relative to the comparison group with a moderate effect size. There was a significant difference between crude and adjusted mean of weight that indicated lose weight was not only related to the effect of theory -based intervention but also it was associated with the mean of weight before intervention as a covariance. The results of a systematic review (10) and a meta-analysis study(46) confirmed using a pedometer in lose weight and body mass reduction index. Although assignment random was used to control covariate variable, there was no equal distribution of marital status variable between two groups, so the effect of this covariate was controlled through statistical method, namely the ANOVA ANCOVA test. Even by controlling the variable of marital status, the pedometer and theory-based intervention significantly increased step-counts and walking and decreased weight in the intervention group compared to the comparison group.

\section{Limitations}

This study was done on women with PCOS referred to Mahdieh hospital, Tehran. Random assignment was used to control selection bias and confounding bias, but the marital status variable was not distributed between the two groups equally, and the researchers had to use stratification in addition to random assignment. The performance bias was possible because the educator was second researcher and taught and followed up the patients of the two groups. In addition, due to lack of similarity of educational intervention in both groups, there was no possibility of blinding, so the control of performance bias was not possible. Another limitation was detection bias. Self-reported PA and cognitive factors influencing PA were measured with the questionnaires. These outcomes were based on reporting of patients (patient report outcome) that increased the error of measuring cognitive outcomes. Therefore, step-counts and weight as objective measures were calculated to control detection bias. The questionnaires and measurements were also performed by someone else outside the research team. To control contamination bias, only the intervention group participated in educational intervention. Training how to use the pedometer and completing the questionnaires were administrated on different days for both groups; so that the patients did not any interact together. To control attrition bias, patients' reasons for not following-up were similar in the two groups, but the percentage of drop out in the comparison group was a little bit higher than the intervention group.

\section{Conclusion}

Identifying individual, social and structural factors associated with PA based on the PRECEDE framework and applying the relevant BCTs boosted pedometer- based programs to increase walking, step counts and diminishing weight of women with PCOS. Therefore, it is suggested BCTs on the basis of a theoretical framework are applied in designing pedometer-based interventions in order to enhance its effectiveness.

\section{Abbreviations}

PCOS: polycystic ovarian syndrome; BCTs: behavioral change techniques, PA: physical activity; IPAQ: International Physical Activity Questionnaire; MET: metabolic equivalents; CVI: content validity index; CVR: content validity ratio.

\section{Declarations}




\section{Acknowledgment}

The authors would like to thank all women who made this study possible.

\section{Funding}

The financial support of this study was provided by Alborz University of Medical Sciences as a research project.

\section{Availability of data and materials}

The datasets generated during the study are available and are attached as supplementary material.

\section{Authors' contributors}

LS, RR were involved in the study design and wrote the proposal. RR implemented intervention and followed the patients. LS conducted the data analysis. RR and LS wrote the first draft of the manuscript. LS and MCHK edited the final manuscript. All authors approved the final manuscript.

\section{Ethics approval and consent to participate}

The study protocol was approved by the ethics committee of the Alborz University of Medical Sciences 2016 (approval No. Abzums. Rec.1395.23). All women participating in the study were informed about the objectives of research and assured about the confidentiality of their information. In addition, informed written consent forms were completed by participants in both groups. This study protocol was registered in the Iranian Registry of Clinical Trials as IRCT20161116030923N3. In order to comply with ethical consideration, the comparison group was benefited from theory-based intervention at the end of the study.

\section{Declaration of interest}

The authors report no conflicts of interest. The authors alone are responsible for the content and writing of the article.

\section{Consent for publication}

Not applicable.

\section{Author details}

1,2. Department of Health Education \& Promotion, Alborz University of Medical Sciences, Karaj, Iran. ${ }^{3}$.Department of Public Health, Mazandaran University of Medical Sciences, Mazandaran, Iran.

\section{References}

1. McBreairty LE, Chilibeck PD, Gordon JJ, Chizen DR, Zello GA. Polycystic ovary syndrome is a risk factor for sarcopenic obesity: a case control study. BMC endocrine disorders. 2019;19(1):70.

2. Legro RS, Arslanian SA, Ehrmann DA, Hoeger KM, Murad MH, Pasquali R, et al. Diagnosis and treatment of polycystic ovary syndrome: an Endocrine Society clinical practice guideline. The Journal of Clinical Endocrinology \& Metabolism. 2013;98(12):4565-92.

3. Sam S. Obesity and polycystic ovary syndrome. Obesity management. 2007;3(2):69-73.

4. Tehrani FR, Simbar M, Tohidi M, Hosseinpanah F, Azizi F. The prevalence of polycystic ovary syndrome in a community sample of Iranian population: Iranian PCOS prevalence study. Reproductive Biology and Endocrinology. 2011;9(1):39.

5. Azziz R, Marin C, Hoq L, Badamgarav E, Song P. Health care-related economic burden of the polycystic ovary syndrome during the reproductive life span. The Journal of Clinical Endocrinology \& Metabolism. 2005;90(8):4650-8.

6. Boyle J, Brennan L, Brinkworth G, Brown W, Burger H, Clarke I, et al. Evidence-based guideline for the assessment and management of polycystic ovary syndrome A single. 2011.

7. Teede HJ, Misso ML, Costello MF, Dokras A, Laven J, Moran L, et al. Recommendations from the international evidence-based guideline for the assessment and management of polycystic ovary syndrome. Human Reproduction. 2018;33(9):1602-18.

8. Lamb JD, Johnstone EB, Rousseau J-A, Jones CL, Pasch LA, Cedars MI, et al. Physical activity in women with polycystic ovary syndrome: prevalence, predictors, and positive health associations. American journal of obstetrics and gynecology. 2011;204(4):352. e1-. e6. 
9. Shishehgar F, Tehrani FR, Mirmiran P, Hajian S, Baghestani AR, Moslehi N. Factors influencing physical activity in women with polycystic ovary syndrome in comparison to eumenorrheic non hirsute women. Glob J Health Sci. 2016;8(10):56382.

10. Bravata DM, Smith-Spangler C, Sundaram V, Gienger AL, Lin N, Lewis R, et al. Using pedometers to increase physical activity and improve health: a systematic review. Jama. 2007;298(19):2296-304.

11. Excellence NIfC. A rapid review of the effectiveness of pedometer interventions to promote physical activity in adults. London: National Institute for Clinical Excellence; 2006.

12. Bassett D. How many steps/day are enough? Preliminary pedometer indices for public health. Sports Med. 2004;34:1-8.

13. Aghdam FB, Moghaddam MHB, Jafarabadi MA, Allahverdipour H, Nikookheslat SD, Nourizadeh R. Explaining the role of personal, social and physical environment factors on employed women's physical activity: a structural equation analysis. Global journal of health science. 2013;5(4):189.

14. Bartholomew LK, Parcel GS, Kok G, Gottlieb NH, Fernandez ME. Planning Health Promotion Programs: An Intervention Mapping Approach. 3rd ed: John Wiley \& Sons P\&T; 2011. 768 p.

15. Green L, Kreuter M. Health Program Planning: An educational and ecological approach. edition. San Francisco, CA, McGraw-Hill; 2005.

16. Michie S, Richardson M, Johnston M, Abraham C, Francis J, Hardeman W, et al. The behavior change technique taxonomy ( 1 ) of 93 hierarchically clustered techniques: building an international consensus for the reporting of behavior change interventions. Annals of behavioral medicine. 2013;46(1):81-95.

17. Sabzmakan L, Mohammadi E, Morowatisharifabad MA, Afaghi A, Naseri MH, Mirzaei M. Environmental determinants of cardiovascular diseases risk factors: a qualitative directed content analysis. Iranian Red Crescent Medical Journal. 2014;16(5).

18. Sabzmakan L, Morowatisharifabad MA, Mohammadi E, Mazloomy-Mahmoodabad SS, Rabiei K, Naseri MH, et al. Behavioral determinants of cardiovascular diseases risk factors: A qualitative directed content analysis. ARYA atherosclerosis. 2014;10(2):71.

19. Sabzmakan L, Jafarabadi MA, Springer A, Morowatisharifabad MA, Mohammadi E. Physical Activity and Healthy Eating Promotion among Adults with Cardiovascular Metabolic Risk Factors: An Application of Intervention Mapping Framework. Health Scope. 2017;6(3).

20. Davies P, Walker AE, Grimshaw JM. A systematic review of the use of theory in the design of guideline dissemination and implementation strategies and interpretation of the results of rigorous evaluations. Implementation Science. 2010;5(1):14.

21. Schulz KF, Altman DG, Moher D. CONSORT 2010 statement: updated guidelines for reporting parallel group randomised trials. BMC medicine. 2010;8(1):18.

22. Randomization. com 2017 [

23. Baker G, Gray SR, Wright A, Fitzsimons C, Nimmo M, Lowry R, et al. The effect of a pedometer-based community walking intervention" Walking for Wellbeing in the West" on physical activity levels and health outcomes: a 12-week randomized controlled trial. International Journal of Behavioral Nutrition and Physical Activity. 2008;5(1):44.

24. Faghri PD, Omokaro C, Parker C, Nichols E, Gustavesen S, Blozie E. E-technology and pedometer walking program to increase physical activity at work. The journal of primary prevention. 2008;29(1):73-91.

25. Committee IR. Guidelines for data processing and analysis of the International Physical Activity Questionnaire (IPAQ)-short and long forms. http://www ipaq ki se/scoring pdf. 2005.

26. Craig CL, Marshall AL, Sjöström M, Bauman AE, Booth ML, Ainsworth BE, et al. International physical activity questionnaire: 12-country reliability and validity. Medicine \& science in sports \& exercise. 2003;35(8):1381-95.

27. Moghaddam MB, Aghdam FB, Jafarabadi MA, Allahverdipour H, Nikookheslat SD, Safarpour S. The Iranian Version of International Physical Activity Questionnaire (IPAQ) in Iran: content and construct validity, factor structure, internal consistency and stability. World applied sciences journal. 2012;18(8):1073-80.

28. Glanz K, Rimer BK, Viswanath K. Health behavior and health education: theory, research, and practice: John Wiley \& Sons; 2008.

29. Waltz C, Strickland O, Lenz E. Measurement in Nursing and Health Research. Springer Publishing Company. New York, NY. 2010.

30. Kok G, Gottlieb NH, Peters G-JY, Mullen PD, Parcel GS, Ruiter RA, et al. A taxonomy of behaviour change methods: an Intervention Mapping approach. Health psychology review. 2016;10(3):297-312.

31. Cohen J. Statistical power analysis for the behavioral sciences: Routledge; 2013.

32. Gourlan M, Bernard P, Bortolon C, Romain A, Lareyre O, Carayol M, et al. Efficacy of theory-based interventions to promote physical activity. A meta-analysis of randomised controlled trials. Health psychology review. 2016;10(1):50-66.

33. Prestwich A, Sniehotta FF, Whittington C, Dombrowski SU, Rogers L, Michie S. Does theory influence the effectiveness of health behavior interventions? Meta-analysis. Health Psychology. 2014;33(5):465.

Page 11/14 
34. Tang MY, Smith DM, Mc Sharry J, Hann M, French DP. Behavior Change Techniques Associated With Changes in Postintervention and Maintained Changes in Self-Efficacy For Physical Activity: A Systematic Review With Meta-analysis. Annals of Behavioral Medicine. 2018.

35. Michie S, Abraham C, Whittington C, McAteer J, Gupta S. Effective techniques in healthy eating and physical activity interventions: a meta-regression. Health Psychology. 2009;28(6):690.

36. Shilts MK, Horowitz M, Townsend MS. Goal setting as a strategy for dietary and physical activity behavior change: a review of the literature. American Journal of Health Promotion. 2004;19(2):81-93.

37. Samdal GB, Eide GE, Barth T, Williams G, Meland E. Effective behaviour change techniques for physical activity and healthy eating in overweight and obese adults; systematic review and meta-regression analyses. International Journal of Behavioral Nutrition and Physical Activity. 2017;14(1):42.

38. Moon D-H, Yun J, Beamer J. The effects of goal commitment on physical activity in adults. Age (years). 2017;54(6.45):54.38-6.15.

39. Morrison JD, Stuifbergen AK. Outcome expectations and physical activity in persons with longstanding multiple sclerosis. The Journal of neuroscience nursing: journal of the American Association of Neuroscience Nurses. 2014;46(3):171.

40. Lutes LD, Steinbaugh EK. Theoretical models for pedometer use in physical activity interventions. Physical therapy reviews. 2010;15(3):143-53.

41. Smith GL, Banting L, Eime R, O'Sullivan G, Van Uffelen JG. The association between social support and physical activity in older adults: a systematic review. International Journal of Behavioral Nutrition and Physical Activity. 2017;14(1):56.

42. Kramer J-N, Kowatsch T. Using Feedback to Promote Physical Activity: The Role of the Feedback Sign. Journal of medical internet research. 2017;19(6):e192.

43. Jirathananuwat A, Pongpirul K. Promoting physical activity in the workplace: A systematic meta-review. Journal of occupational health. 2017:16-0245-RA.

44. Sniehotta FF, Scholz U, Schwarzer R. Action plans and coping plans for physical exercise: A longitudinal intervention study in cardiac rehabilitation. British journal of health psychology. 2006;11(1):23-37.

45. Pal S, Cheng C, Egger G, Binns C, Donovan R. Using pedometers to increase physical activity in overweight and obese women: a pilot study. BMC Public Health. 2009;9(1):309.

46. Cai X, Qiu S, Yin H, Sun Z, Ju C, Zügel M, et al. Pedometer intervention and weight loss in overweight and obese adults with Type 2 diabetes: a meta-analysis. Diabetic Medicine. 2016;33(8):1035-44.

\section{Figures}




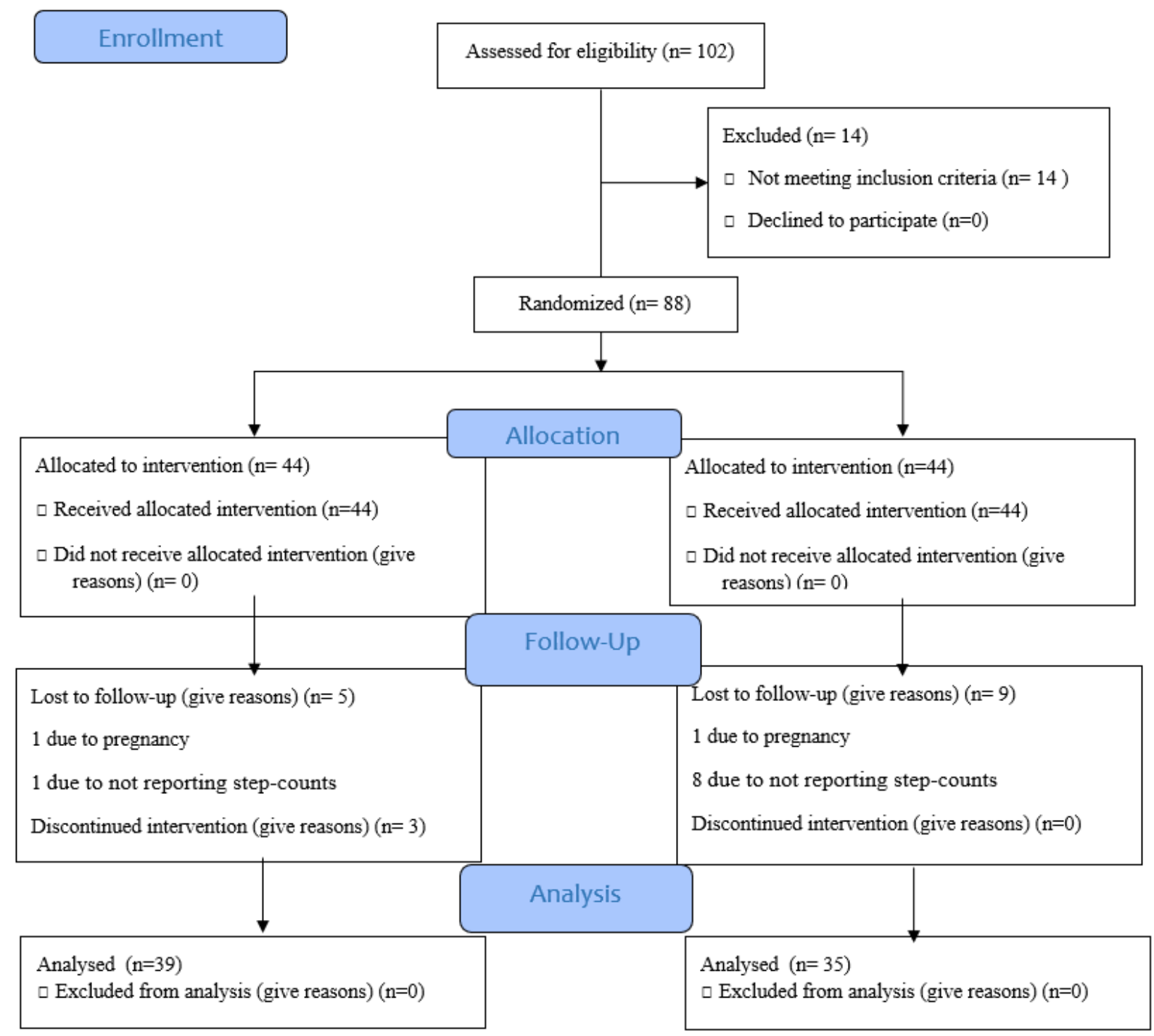

Figure 1

The flow diagram of participants through the study 


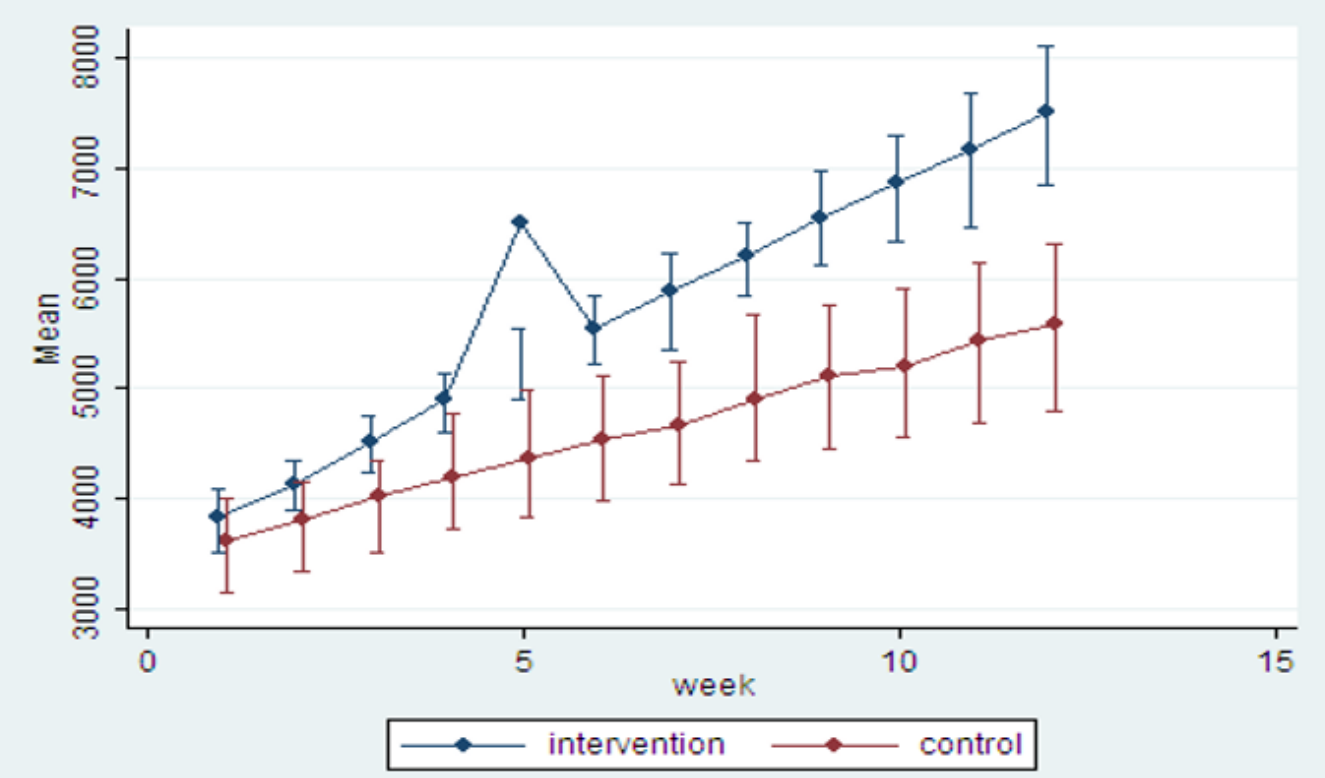

\section{Figure 2}

The mean steps/week for the two groups over 12 weeks

\section{Supplementary Files}

This is a list of supplementary files associated with this preprint. Click to download.

- consort2010checklist.doc

- DataPCOS.sav 